\title{
CARACTERÍSTICAS CLÍNICO QUIRÚRGICAS DE PACIENTES DE 75 AÑOS O MÁS COLOSTOMÍZADOS EN EL HOSPITAL NACIONAL EDGARDO REBAGLIATI MARTINS
}

\author{
CLINICAL AND SURGICAL CHARACTERISTICS OF PATIENTS FROM 75 YEARS OR OLDER \\ COLOSTOMIZED AT HOSPITAL EDGARDO REBAGLIATI MARTINS \\ Carlos Jhonatan Lobato-Jeri',a, Magdiel José Manuel Gonzales-Menéndez ${ }^{1,2, a, b, c,}$, Cecilia Roxana Salinas-Salas 1,a,d,e
}

\begin{abstract}
RESUMEN
Objetivos: Determinar las características clínicoquirúrgicas de los pacientes de 75 o más años colostomizados en el Servicio de Cirugía de Colon, Recto y Ano del Hospital Nacional Edgardo Rebagliati Martins de enero 2013 a diciembre del 2014. Material y métodos : Estudio transversal en el que se revisó las historias clínicas del servicio de Cirugía de Colon, Recto y Ano desde enero de 2013 hasta diciembre de 2014. La población de estudio estuvo constituida por 38 pacientes con edades de 75 años o más, sometidos a Colostomía según los criterios de inclusión y exclusión establecidos, Los datos se ingresaron en una base de SPSS para su análisis. Resultados: La edad media fue de 81.37 años. 63.2 $\%$ de pacientes tuvo de 75 a 80 años; $21.1 \%$, de 81 a 90 y $15.8 \%$, más de 90 años. $73.7 \%$ era de sexo femenino. $81.3 \%$ tenía Hipertensión arterial; $18.8 \%$, Diabetes mellitus 2; igual que Enfermedad renal crónica. $43.8 \%$ había sido operado previamente en Abdomen o Pelvis y $62.5 \%$ tenía una clasificación ASA III o IV. $42.11 \%$ de pacientes fue colostomizado por Cáncer colorrectoanal irresecable, única causa de Obstrucción intestinal en estos pacientes; mientras que el $21.05 \%$, como parte de una Resección abdominoperineal. El $68.42 \%$ de Colostomías fue definitiva y el $52.63 \%$, terminal. $52.63 \%$ de pacientes presentó complicaciones postoperatorias: Shock séptico (15.79\%), Infección de sitio operatorio (10.53\%), Necrosis colostómica (5.26\%), Abscesos intraabdominales (5.26 \%) y otras (15.79\%). El $52.63 \%$ de complicados se distribuyó, según grupo etario: $26.32 \%, 10.53 \%$ y $15.79 \%$ para los grupos de $75-80,81$ - 90, y más de 90 años, mientras que el $47.37 \%$ sin complicaciones, en $36.84 \%, 10.53 \%$ y $0 \%$, respectivamente. Los pacientes que tenían una clasificación ASA III o IV se complicaron el doble que los que no la tenían. Conclusión: El paciente mayor o igual de 75 años, operado de colostomía, es, en su mayoría, del grupo etario de 75 a 80 años, de sexo femenino, operado de obstrucción intestinal por Cáncer colorrectoanal irresecable, con Colostomía definitiva, terminal, con alto riesgo de complicarse, probablemente en relación a una elevada clasificación ASA.
\end{abstract}

Palabras clave: Colostomía; Características clinicoquirúrgicas (fuente: DeCS BIREME).

\begin{abstract}
Objective: To determine the clinical and surgical characteristics of patients aged 75 and older who underwent colostomy in the department of Surgery of Colon, Rectum and Anus of "Edgardo Rebagliati Martins National Hospital" during January 2013 to December 2014. Materials and methods: Transversal study where clinical records from the Colon, Rectum and Anus Surgery Service were reviewed from January 2013 to December 2014. The study population was set up by 38 patients aged 75 or more. They were subject to colostomy according to inclusion and exclusion established criteria. Data was registered in a SPSS database for its analysis (IBM). Results: The average age was 81.37 years. $63.2 \%$ of patients had 75 to $80 ; 21.1 \%$, from 81 to 90 and $15.8 \%$ over 90 years. $73.7 \%$ were female. $81.3 \%$ had high blood pressure; $18.8 \%$, Diabetes mellitus 2 ; As well as chronic kidney disease. $43.8 \%$ had been previously undergone surgery in abdomen or pelvis and $62.5 \%$ had a classification of ASA III or IV. $42.11 \%$ of patients underwent colostomy because of unresectable colon, rectum or anus cancer, sole cause of intestinal obstruction in these patients; while $21.05 \%$ as part of an AP resection. $68.42 \%$ of colostomies were final and $52.63 \%$ of them were terminal. $52.63 \%$ of patients had postoperative complications: septic shock (15.79\%), surgical site infection (10.53\%), colostomy necrosis (5.26\%), intra-abdominal abscesses (5.26\%) and others (15.79\%). The 52.63\% of complicated cases were distributed according to age group: $26.32 \%$, $10.53 \%$ and $15.79 \%$ for groups of $75-80,81-90$, and over 90 years, while $47.37 \%$ without complications were distributed $36.84 \%, 10.53 \%$ and $0 \%$, respectively. Patients who had a classification of ASA III or IV doubled those without complications. Conclusion: The patient aged $\geq 75$, who underwent colostomy, is mostly in the age group 75-80 years old, female, operated because of intestinal obstruction (colorectoanal unresectable cancer), with a clear and terminal colostomy, and has high risk for complications, probably related to high ASA classification.
\end{abstract}

Key words: Colostomy, Clinical and surgical charateristics (source: MeSH NLM).

${ }^{1}$ Facultad de Medicina Humana, Universidad Ricardo Palma, Lima, Perú.

${ }^{2}$ Instituto de Investigación en Ciencias Biomédicas, Facultad de Medicina Humana, Universidad Ricardo Palma, Lima, Perú.

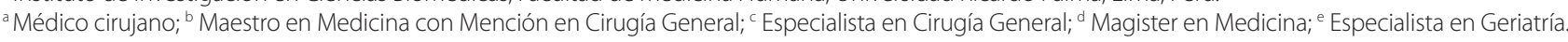
Correspondencia: Magdiel José Manuel Gonzales Menéndez. Dirección: Av. Benavides 5440, Surco, Lima, Perú. Universidad Ricardo Palma. Teléfono: $708-0000$. Anexo: 6011. Correo : magdiel.gonzalesm@urp.pe

Citar como: Carlos Jhonatan Lobato-Jeri, Magdiel José Manuel Gonzales-Menéndez, Cecilia Roxana Salinas-Salas. Características clínico quirúrgico de pacientes de 75 años o más colostomízados en hospital nacional Edgardo Rebagliati Martins [Artículo Original]. Rev. Fac. Med. Hum. 2016;16(2):39-45. DOI 10.25176/RFMH.v16.n2.667 


\section{INTRODUCCIÓN}

Desde tiempos antiguos, pasando por la realización de la primera Colostomía programada exitosa, en 1973, por Duret, a nivel iliaco, a un recién nacido de 3 días de vida con ano imperforado, viviendo el paciente por 45 años luego de la cirugía; hasta la actualidad, la teoría ha evolucionado, llevando consigo adelantos en cuando a eficacia y sobrevida con menos complicaciones, hasta nuestros tiempos' ${ }^{1}$.

El hombre ha evolucionado junto con el conocimiento, sobreviviendo más. En la edad de piedra, el hombre vivía un promedio de 19 años; en el siglo XVI alcanzaba los 27 años; en 1900 solo los 40 años; en 1945 aumentó a los 57 años; en 1970 ya alcanzaba los 60 años; $y$, actualmente, en nuestro medio, alcanza los $74^{1}$ años de esperanza de vida; y este crecimiento etario camina de la mano con la búsqueda de mejor salud para este grupo, para con esta mejor salud, siga aumentando la esperanza de vida².

La población geriátrica es más susceptible fisiológicamente a complicaciones y formas más severas y atípicas de las diversas patologías. Por lo que en los inicios de la cirugía a dichos pacientes se les trataba de manera paliativa en vez de curativa, actitud que ha ido cambiando a través de los años, en gran medida, por el avance en el conocimiento nuevas razones, distintas de la edad, para explicar la morbilidad. Es así que muchos estudios avalan la relevancia de las comorbilidades y la dependencia del anciano por encima de la edad misma, como responsables, en el ámbito quirúrgico, de la morbimortalidad postoperatoria, siendo la edad, en muchos casos, no importante en la agresividad y efecto terapéuticos; y siguiendo este razonamiento, se inició la búsqueda de nuevas mediciones y escalas que incluyeran o excluyeran a la edad, con el objetivo de acercarse más a una mejor decisión operatoria ${ }^{2,3}$.

La patología colorrectal es de gran importancia en el adulto mayor $y$, dentro de ella, la exteriorización del tránsito intestinal mediante una colostomía es de las técnicas más usadas, tanto aislada como parte de una cirugía mayor, dado el efecto principalmente para tratar la opstructicción'.

Conociendo sobre colostomías en el adulto mayor, conoceremos mucho más sobre la patología colon, recto y anal en ellos. Conociendo sobre esta, conoceremos sobre la cirugía en el anciano. Y conociendo sobre esta última, estaremos mejor preparados para afrontar de manera clara y menos subjetiva, las necesidades del paciente.

\section{MATERIAL Y MÉTODOS}

Se realizó un trabajo transversal con una población de pacientes de 75 años o más, sometidos a Colostomía en el Servicio de Cirugía de Colon, Recto y Ano del Hospital Nacional Edgardo Rebagliati Martins (HNERM) localizado en el distrito de Jesús María, departamento de Lima, Perú; de enero 2013 a diciembre del 2014 y que cumplieran con los siguientes criterios de inclusión y exclusión:

\section{Criterios de inclusión}

- Pacientes con edad igual o mayor a 75 años cumplidos, ingresados al servicio durante el período mencionado, sometidos a intervención quirúrgica que incluyera, como base o complemento de la misma, la realización de colostomía.

- Pacientes sometidos a intervención quirúrgica que incluyese la realización de una Colostomía, en el servicio de emergencia de adultos durante el periodo mencionado, por médicos asistentes del servicio de cirugía de colon, recto y ano, y que, para el manejo postoperatorio, ingresaron al servicio.

- Pacientes hospitalizados en el servicio y sometidos a una cirugía diferente o que no incluyera colostomía, que fueron reoperados, realizándoseles una colostomía, por motivos inherentes a complicaciones de la cirugía primaria o de extensión de la enfermedad de base.

- Pacientes hospitalizados en otros servicios, que durante su hospitalización fueron intervenidos, siendo sometidos a colostomía electiva o de emergencia, a cargo de médicos asistentes del servicio de cirugía de colon, recto y ano.

\section{Criterios de exclusión}

- Pacientes colostomizados por médicos asistente del Servicio de cirugía de colon, recto y ano, y que, para el manejo postoperatorio, ingresaron a otros servicios de Cirugía General

- Pacientes colostomizados por médicos asistentes de otros servicios, aunque ingresaran al Sevicio luego para el manejo postoperatorio

- Pacientes con Historias clínicas incompletas

- Pacientes con Historias clínicas no ubicables

- Pacientes fallecidos en el periodo intraoperatorio

Se elaboró una ficha de recolección de la información que contiene las variables que permitirán cumplir con los objetivos planteados. La fuente de información fue de carácter indirecta, a través de 
revisión de expedientes clínicos que cumplan los criterios de inclusión.

A fin de garantizar los aspectos éticos en la investigación se manejaron todos los datos confidencialmente y no se consignarán los nombres de los pacientes para evitar su identificación.

Para el procesamiento y elaboración de gráficas de resultados, tablas de doble entrada (distribución de frecuencias), medidas de tendencia central (media), y recuento en valores absolutos y porcentajes se utilizó el software SPSS 23.

\section{RESULTADOS}

Fueron sometidos a colostomía 128 pacientes, de los cuales 38 tenían 75 años de edad o más (29.7 $\%)$, que constituyó la población de estudio. La edad media fue 81.37 años.

\section{Características pre quirúrgicas}

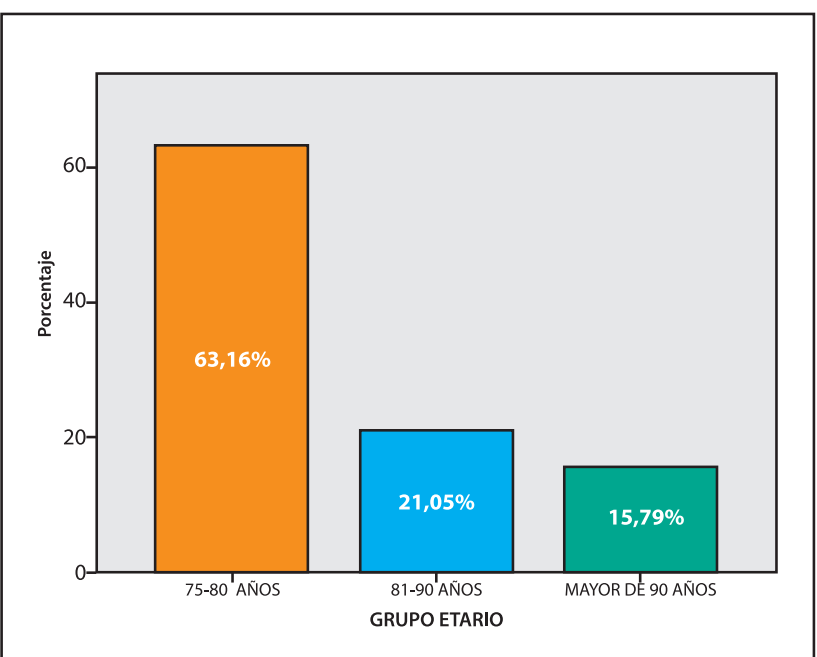

Gráfica 1. Distribución de pacientes según grupo etario.

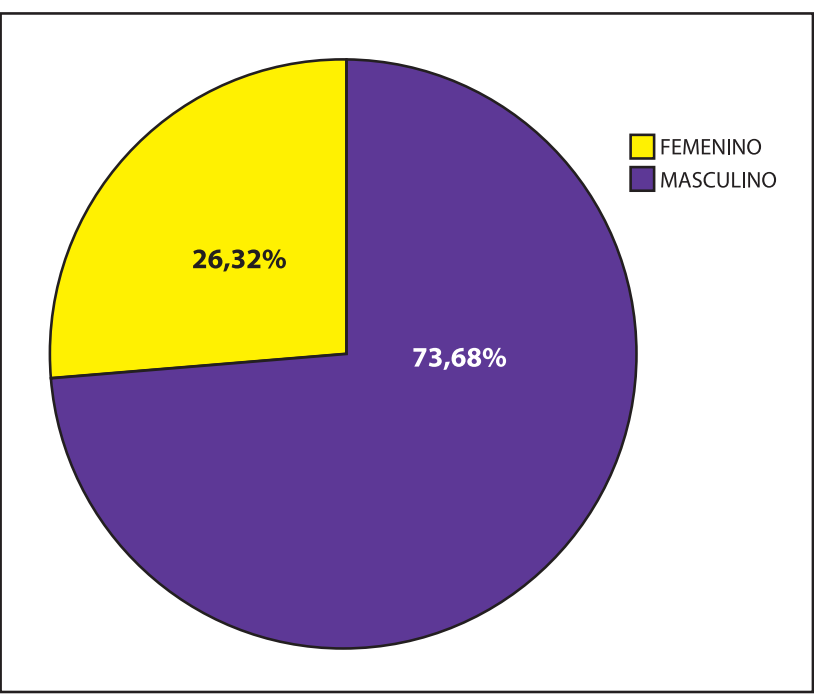

Gráfica 2. Distribución de pacientes según sexo.
Tabla 1. Distribución de pacientes según comorbilidades.

\begin{tabular}{|c|c|c|c|c|}
\hline & \multicolumn{2}{|c|}{ Respuestas } & \multirow{2}{*}{$\begin{array}{c}\text { Porcentaje } \\
\text { de casos }\end{array}$} \\
\hline & & $\mathbf{N}^{\circ}$ & Porcentaje & \\
\hline \multirow[t]{5}{*}{ Antecedentes ${ }^{a}$} & $\begin{array}{l}\text { Hipertensión } \\
\text { Arterial }\end{array}$ & 26 & $36,1 \%$ & $81,3 \%$ \\
\hline & $\begin{array}{l}\text { Diabetes } \\
\text { Mellitus } \\
\text { Tipo } 2\end{array}$ & 6 & $8,3 \%$ & $18,8 \%$ \\
\hline & $\begin{array}{l}\text { Enfermedad } \\
\text { Renal Crónica }\end{array}$ & 6 & $8,3 \%$ & $18,8 \%$ \\
\hline & $\begin{array}{l}\text { Cirugías } \\
\text { Abdomi- } \\
\text { nopélvicas } \\
\text { Previas }\end{array}$ & 14 & $19,4 \%$ & $43,8 \%$ \\
\hline & $\begin{array}{l}\text { Clasificación } \\
\text { Asa III-IV }\end{array}$ & 20 & $27,8 \%$ & $62,5 \%$ \\
\hline Total & & 72 & $100,0 \%$ & $225,0 \%$ \\
\hline
\end{tabular}

\section{Características pre quirúrgicas}

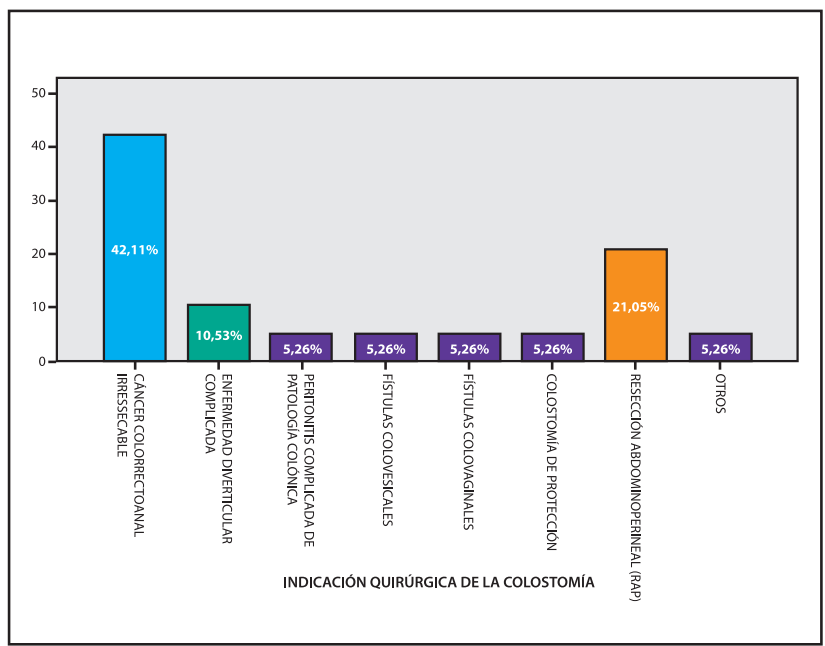

Gráfica 3. Distribución de pacientes según indicación quirúrgica.

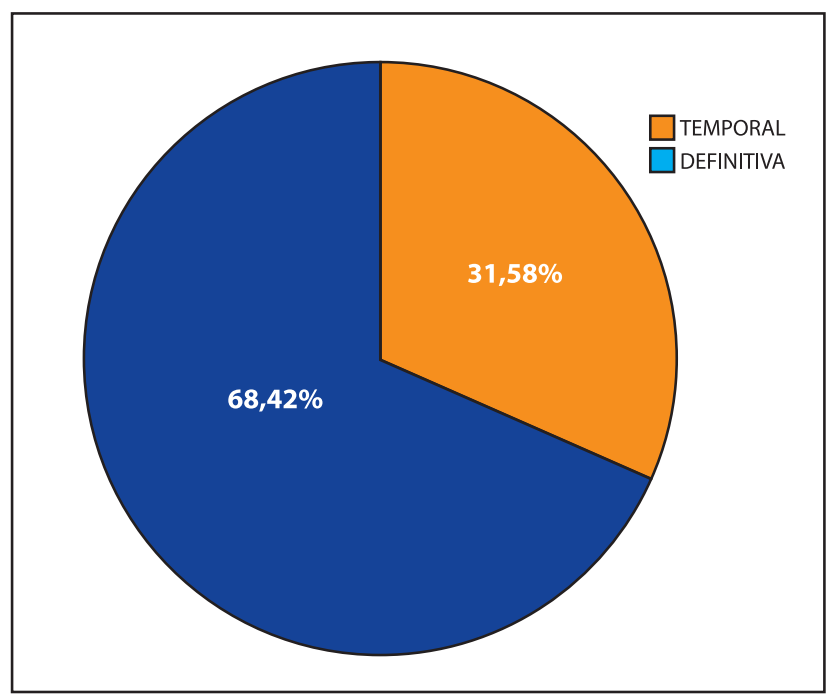

Gráfica 4. Distribución de pacientes según tipo de colostomía. 


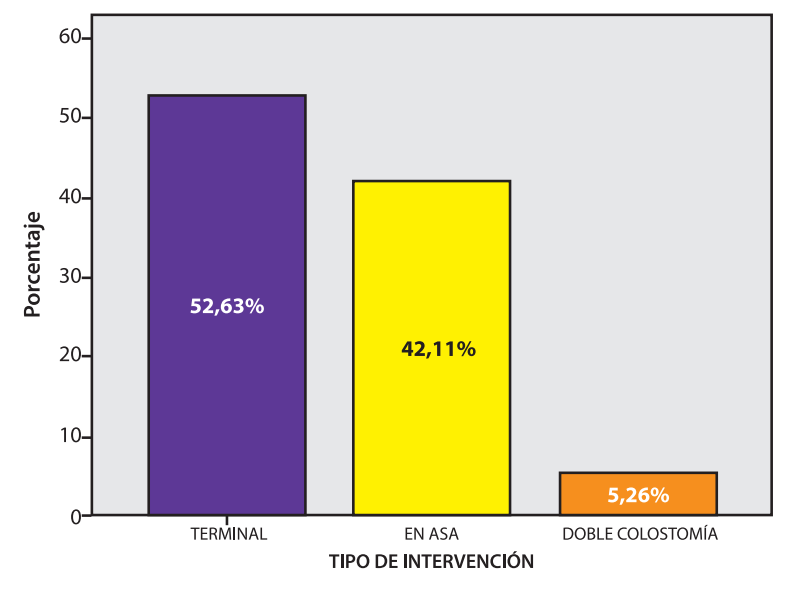

Gráfica 5. Distribución de pacientes según tipo de intervención.

\section{Características postquirúrgicas}

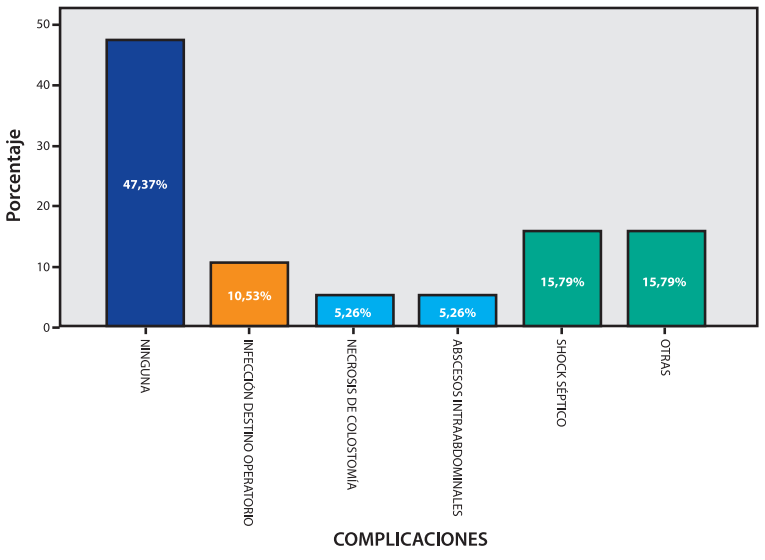

Gráfica 6. Distribución de pacientes según complicaciones.

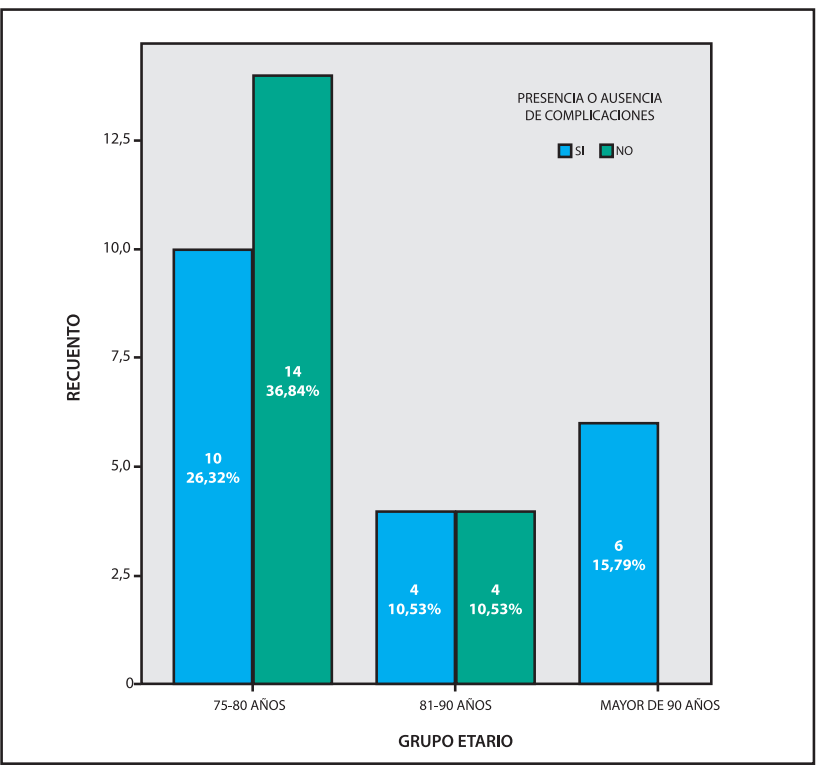

Gráfica 7. Distribución de presencia o ausencia de complicaciones según grupo etario.

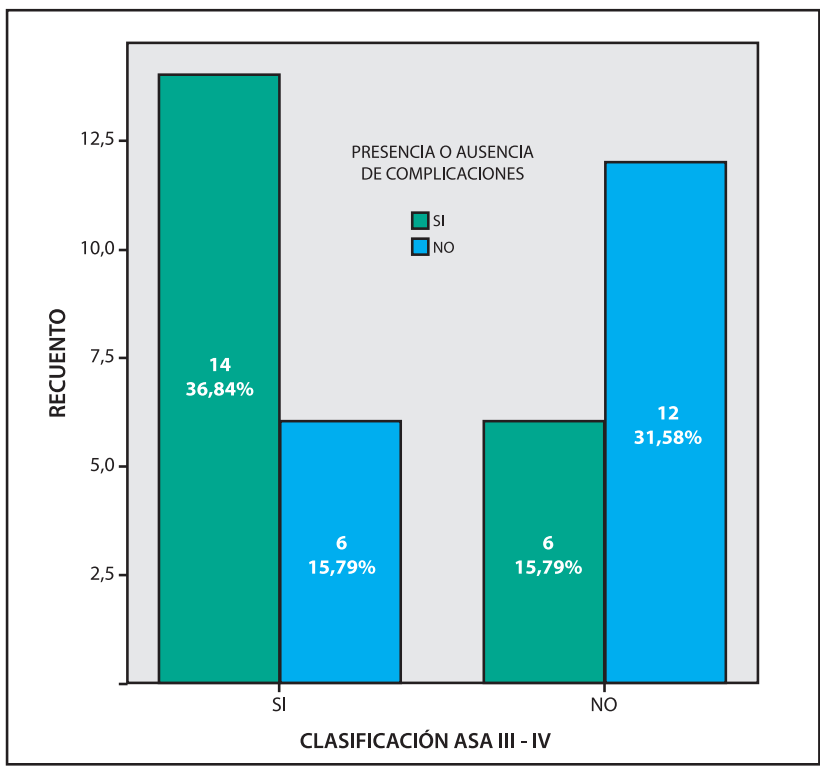

Gráfica 8. Distribución de presencia o ausencia de complicaciones según presencia o ausencia de clasificación asa III o IV.

\section{DISCUSIÓN}

La edad media de los pacientes estudiados fue de 81.37 años, distribuidos en el grupo etario de 75 a 80 años, lo que se encuentra en relación a la esperanza de vida en nuestro país que, según datos del 2014 del Instituto Nacional de Estadística e Informática (INEI) es de 74.1 años'.

Con respecto al sexo, se evidencia un notorio predominio femenino $(73.68 \%)$, lo que contrasta con la mayoría de estudios. Sanjinés Ticona, en Tacna (2015) o Morales y col., en Cusco, Seok In Seo (Corea del Sur, 2013) y Bouassida (Tunes, 2015) encontraron importante mayoría masculina en operados de patología colorrectal; sustentada, en lo referente a Colostomías, sobre todo, en la mayor frecuencia masculina en padecimientos como Obstrucción intestinal. Existen estudios con predominio femenino, como el de Leal de Alencar, en Brasil (2014), lo que se asocia a la incidencia creciente de Cáncer colorrectal en mujeres ${ }^{2,3,4,5,6}$.

La Hipertensión aparece como la más frecuente (81.3\%) de la comorbilidades, muy por encima de otras enfermedades crónicas, mientras que $31.58 \%$ de pacientes no padecía enfermedades crónicas. Esto difiere de lo reportado por Sanjinéz Ticona y col., en el Hospital Hipólito Unanue, Tacna (2015), 93.1\% sin antecedentes patológicos, siendo la Diabetes mellitus la única enfermedad presente (6.9\%); hallazgos similares a los de Morales y col., con $95.65 \%$ de pacientes sin comorbilidades. Si 
bien es cierto, en el primero de ambos estudios, la población predominante fue joven (21-40 años, con $37.9 \%)$, un porcentaje no muy lejano tenía de 61 a 80 años de edad (31.0\%), mientras que, en el segundo estudio señalado, la población predominante si fue la geriátrica (61-70 años, 28\%); por lo que deben considerarse otros motivos para tales diferencias en la proporción comorbilidad / no comorbilidad, como el nivel de atención (por ejemplo, el Hospital Hipólito Unanue es un establecimiento de atención referencial especializada, nivel II-2, MINSA; mientras que el HNERM es de categoría III-1, nivel IV de EsSalud), lo que deriva en mayor número de pacientes con comorbilidades ${ }^{2,3}$.

El $62.5 \%$ de pacientes estuvo concentrado en los niveles III o IV de la clasificación ASA (American Society of Anesthesiologists). Bouassida y col., encontraron que el $48.4 \%$ contaba con una clasificación ASA III o IV (en mayores de 70 años) así como en el 2010 en Grecia, Kesisoglou y col. en su estudio en mayores de 70 años, encontraron que $33.3 \%$ contaban con una clasificación ASA III o IV. Recuentos considerables, aunque menores, al del presente estudio, considerando los 5 años de margen de diferencia en las edades mínimas de aquellos estudios con el que ahora se discute. Una elevada frecuencia de pacientes con clasificación ASA II o IV podría asociarse, como las comorbilidades, al nivel de atención del establecimiento de salud ${ }^{5,7,8}$.

Con respecto a la indicación quirúrgica, se observa claro predominio por parte de la Obstrucción intestinal, representada de manera absoluta por el cáncer colorrectoanal irresecable $(42.11 \%)$, seguida por el cáncer de recto inferior o Ano resecables (con la Resección abdominoperineal como planteamiento quirúrgico); de esto deriva que son las neoplasias malignas colorrectoanales las responsables de la amplia mayoría de colostomías (en total, 63.16\%), detalle que pone evidencia el carácter netamente oncológico del servicio de Cirugía de Colon, Recto y Ano del establecimiento de salud en estudio. Morales y col., en Cusco, hallaron, también, predominio de cáncer colorrectal como indicación de colostomías $(52,78 \%)$, seguido del vólvulo de Sigmoides (27.78\%), del que no hubo casos en el presente estudio. Ambos resultados contrastan con lo reportado por Sanjinéz Ticona, en Tacna (MINSA), ya que, a pesar de predominar la patología obstructiva (44.8\%), dentro de ella, como principal motivo quirúrgico, se encontró al Vólvulo de Sigmoides (46.2\% de los cuadros obstructivos), relegando al Cáncer de Colon a secundarlo (30.8\%), seguidos de la patología inflamatoria. La razón de estas distribuciones deviene de la orientación de cada servicio quirúrgico, aunque resalta, de todos modos, la presencia de patología neoplásica maligna como indicación importante en todos los estudios. Leal de Alencar y col. en su estudio sobre Perfil de ostomizados, encontraron como principal causa patológica asociada a ostomía, al igual que en el presente estudio, a la Neoplasia maligna colorrectal ( $80 \%$ ), sobre todo CoIon descendente - sigmoides y Recto. Siguiendo la misma línea, Pavlidis y col. (población de pacientes mayores de 65 años) y Formijne y col. (edad media en estudio de 67 años) también hallaron mayoría de Colostomías por patología maligna ( $54.17 \%$ y $46 \%$, respectivamente). Todo ello refuerza la idea de que la patología obstructiva, sobre todo cancerosa, se yergue a la cabeza de las indicaciones quirúrgicas colostómicas $3,2,6,9,10$.

Teniendo en cuenta el tipo de colostomía (posibilidad de restitución posterior de la continuidad colónica), la mayoría de Colostomías fueron definitivas (68.42\%), esto se explica por la alta frecuencia de neoplasias malignas irresecables, y por la también alta frecuencia de Resecciones abdominoperineales, que incluyen en su procedimiento, la realización de Colostomía definitiva. Este resultado, si bien aparenta contrastar marcadamente con el $6.9 \%$ de Colostomías definitivas halladas por Sanjinéz y col., no es un detalle menor tener en consideración que los dos pacientes que representan ese porcentaje (de 61 y 65 años de edad, respectivamente) tuvieron el diagnóstico de Cáncer colorrectal, lo que, tomando en cuenta el hecho de que en este estudio no solo se consideraron pacientes geriátricos, podría sostener la idea de mayoría de Colostomías definitivas en pacientes con este último diagnóstico ${ }^{2}$.

Al tomar en consideración el tipo de intervención quirúrgica (tipo de colostomía según morfología), la terminal fue la más frecuente $(52.63 \%)$, debido quizás, de manera similar al resultado previo, a la mayoría de obstrucciones intestinales por Cáncer colorrectoanal irresecable y por resección abdominoperineal. Este predominio de las colostomías terminales coincide con el hallado por Sanjinéz Ticona, con un $72.4 \%$, principalmente por Obstrucción neoplásica y Diverticulitis aguda sigmoidea. Dos pacientes fueron sometidos a doble colostomía, teniendo como diagnóstico Peritonitis complicada de 
patología colónica, por dehiscencia de anastomosis previa, por lo que ambos cabos fueron exteriorizados, para facilitar una próxima restitución de tránsito y limpieza de secreción mucosa por la fístula (cabo distal). No se encontró antecedente de doble Colostomía en la literatura².

Un $52.63 \%$ de pacientes presentó alguna complicación, como principal evento adverso asociado a la cirugía, se encontró al shock séptico (15.79\%, 6 pacientes, todos fallecidos por esa razón) y como principal complicación local, hallamos la Infección de sitio operatorio (10.53\%). Sanjinéz Ticona reporta sólo $16.9 \%$ de complicaciones, la gran mayoría local (Necrosis colostomal como la más frecuente), lo que sustenta, nuevamente, las diferencias en base a nivel de atención en los establecimiento de salud, además de tratarse, este último, de un estudio en población más joven y con menos comorbilidades, por lo que ambas variables, edad y comorbilidades (y probablemente la clasificación ASA), podrían estar asociadas con las complicaciones, por lo que conviene ahora analizar las gráficas que distribuyen complicaciones según grupos etarios y presencia o no de clasificación ASA III o IV².

Analizando la frecuencia de complicaciones según grupo etario, es posible notar como la relación entre pacientes complicados y no complicados se va invirtiendo a medida que aumenta el grupo etario, llegando a complicarse todos los pacientes mayores de 90 años. Esto podría sugerir asociación entre la edad y la presencia de complicaciones; sin embargo, es muy importante mencionar que todos los pacientes con edades mayores a 90 años en el presente estudio, tenían una clasificación ASA III o IV y había mayor frecuencia de pacientes con dicha clasificación de estado físico a medida que se progresaba en el grupo etario. Pavlidis y col. encontraron, en su población de pacientes de 65 años o más sometidos a cirugía colorrectal, $23.8 \%$ de complicaciones, mientras que Hermans y col. en su estudio realizado en Holanda, buscando evaluar el pronóstico de los pacientes mayores de 75 años postoperados de Cáncer de Colon, identificaron $43 \%$ de complicaciones, aunque, al tratarse de un estudio comparativo, pudo evidenciarse que los pacientes menores de 75 años se complicaron en el mismo porcentaje, disminuyendo la relevancia del factor edad en este estudio. Kesisoglou, por su parte, halló $66.67 \%$ de complicaciones dentro del grupo de pacientes mayores de 70 años postoperados de Cán- cer colorrectal frente a un $40 \%$ de complicaciones en menores de esa edad. La disparidad en el recuento de complicaciones en grupos etarios geriátricos, aunque sin tener mismos valores etarios mínimos y máximos, sumado a la similitud de complicaciones en geriátricos o no geriátricos intervenidos por patología Colorrectal en algunos estudios (como el de Hermans) indicaría que no es específicamente la edad, la variable asociada a las complicaciones ${ }^{9,8,11}$.

Finalmente si evaluamos la gráfica №8 (ASA vs. Presencia de complicaciones), llama la atención que, de los 20 pacientes con complicaciones, 14 tenían una clasificación ASA III o IV, y de los 18 no complicados, 12 no tuvieron dicha clasificación ASA. Desde otro enfoque, más cronológico, de los 20 pacientes con clasificación ASA (estado físico preoperatorio) III o IV, 14 se complicaron posteriormente, mientras que, de los 18 pacientes que, antes de ser intervenidos, no tenían tal clasificación, solo 6 se complicaron. El número de pacientes complicados y no complicados es similar, así como los recuentos de pacientes con clasificación ASA III o IV y los que no la tenían (es decir, clasificación ASA I o II), lo que permite mejor visualización y análisis del comportamiento de estas variables. Por todo ello, se podría deducir que la clasificación ASA debería tener relación con la morbilidad postoperatoria, afirmación que debe confirmarse con estudios analíticos. Estos hallazgos son reforzados por los encontrados por Kesisoglou y colaboradores, que señalan que, de los 11 pacientes con clasificación ASA III o IV, 10 padecieron de alguna complicación; mientras que, de los 33 pacientes con una clasificación ASA I o II, 14 se complicó (complicación en grupo con clasificación ASA III o IV de $90.9 \%$, frente a una de $42.4 \%$ en el otro grupo). Este último estudio permite, a la vez, observar que a medida que la clasificación ASA aumenta (del I al IV), la proporción entre complicados y no complicados también lo hace. Si se añade el hecho de que los pacientes fallecidos fueron los 6 con edades mayores a 90 años, todos con clasificación ASA III o IV, podría decirse que la clasificación ASA tiene que ver con morbimortalidad 8 .

Financiamiento: Autofinanciado.

Conflicto de interés: Los autores declaran no tener conflictos de interés en la publicación de este artículo.

Recibido: 07 de Julio de 2016

Aprobado: 10 de Agosto de 2016 


\section{REFERENCIAS BIBLIOGRÁFICAS}

1. Instituto Nacional de Estadística e Informática (INEI) 2014.

2. Sanjinéz M. Frecuencia, indicaciones y complicaciones de las colostomías realizadas en pacientes mayores de 14 años del Servicio de Cirugía del Hospital Hipólito Unanue de Tacna 2006-2011. Tacna; 2015.

3. Morales A, Ezra D, Aedo J, Becerra J. Auditoría en colostomias, ileostomias y otras enterostomias. Rev Situa [Internet]. 1999 [cited 2016 Dic 11];14(7):24-31. Available from: http://sisbib.unmsm.edu.pe/bvrevistas/situa/1999_n14/auditoria.htm.

4. Seo SI, Yu CS, Kim GS, Lee JL, Yoon YS, Kim CW, et al. Characteristics and risk factors associated with permanent stomas after sphincter-saving resection for rectal cancer. World J Surg. 2013;37(10):2490-6.

5. Bouassida M. Surgery for Colorectal Cancer in Elderly Patients: How Could We Improve Early Outcomes ? J Clin Diagnostic Res [Internet]. 2015;9(5):4-9. Available from: http://jcdr.net/article_fulltext.asp?iss$n=0973-709 x \&$ year $=2015$ \& volume $=9$ \&issue $=5$ \&page $=P C 04 \& i s s-$ $n=0973-709 x \& i d=5973$.

6. Leal A, Maria E, Rangel L, Miranda SM. Perfil de pacientes estomizados : revisão integrativa da literatura Profile of ostomy patients : integrative review of the literature Perfil de los pacientes ostomizados: revisión integrativa de la literatura. 2014.

7. American Society of Anesthesiologists physical status scale Last approved by the ASA House of Delegates on October 15, 2014. [cita- do 2016 Dic 11]. Disponible en: http://www.asahq.org/ /media/Sites/ ASAHQ/Files/Public/Resources/standards-guidelines/asa-physical-status-classification-system.pdf.

8. KESISOGLOU I, PLIAKOS I, SAPALIDIS K, DELIGIANNIDIS N, PAPAVRAMIDIS S. Emergency treatment of complicated colorectal cancer in the elderly. Should the surgical procedure be influenced by the factor "age"? Eur J Cancer Care (Engl) [Internet]. 2010;19(6):820-6. Available from: http://doi.wiley.com/10.1111/j.1365-2354.2009.01119.x.

9. Pavlidis TE, Marakis G, Ballas K, Rafailidis S, Psarras K, Pissas D, et al. Safety of bowel resection for colorectal surgical emergency in the elderly. Colorectal Dis [Internet]. 2006;8(8):657-62. Available from: http://www. ncbi.nlm.nih.gov/pubmed/16970575 25.

10. Formijne Jonkers HA, Draaisma WA, Roskott AM, van Overbeeke AJ, Broeders IAMJ, Consten ECJ. Early complications after stoma formation: a prospective cohort study in 100 patients with 1-year follow-up. Int J Colorectal Dis [Internet]. 2012;27(8):1095-9. Available from: http://link. springer.com/10.1007/s00384-012-1413-y 4.

11. Hermans E, van Schaik PM, Prins HA, Ernst MF, Dautzenberg PJL, Bosscha K. Outcome of colonic surgery in elderly patients with coIon cancer. J Oncol [Internet]. 2010;2010(January 2004):865908. Available from: http://www.scopus.com/inward/record.url?ei$d=2-s 2.0-77954637075 \&$ partnerID=tZOtx3y1.

Consulte la Versión Electrónica de la Revista:

Facultad de Medicina Humana Universidad Ricardo Palma

http://revistas.urp.edu.pe/ojs/index.php/RFMH
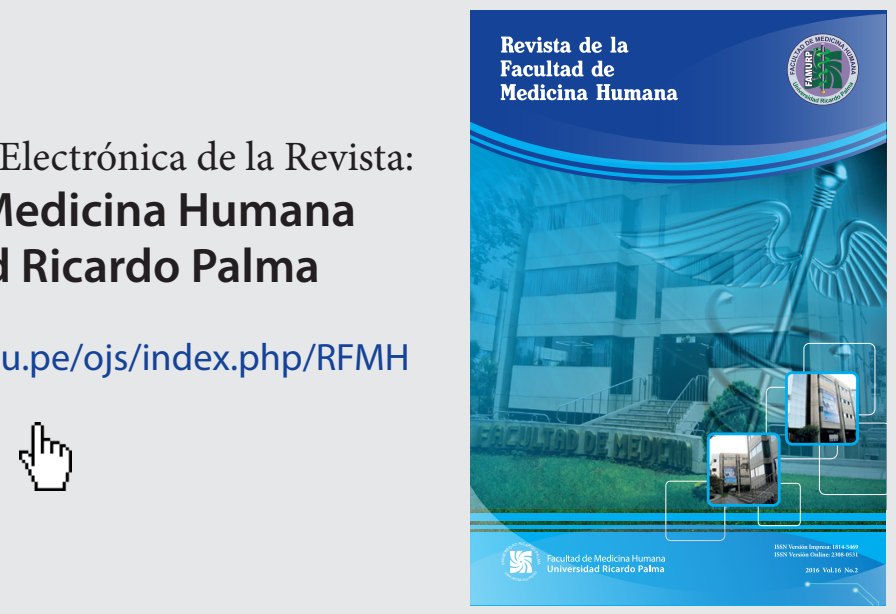